\title{
Stochastic modelling of thermal effects on a ferromagnetic nano particle
}

\author{
Stéphane Labbé* and Jérôme Lelong ${ }^{\dagger}$ \\ Laboratoire Jean Kuntzmann, Université Grenoble Alpes, FRANCE.
}

October 30, 2018

\begin{abstract}
In this work, we are interested in the behaviour of a single ferromagnetic mono-domain particle submitted to an external field with a stochastic perturbation. This model is a step toward the mathematical understanding of thermal effects on ferromagnets. In a first part, we discuss modelling issues and propose several ways to integrate a random noise in the deterministic model. Then, among all these approaches, we focus on the more natural one and study its long time behaviour. We prove that the system converges to the unique stable equilibrium of the deterministic model and make precise the $L^{p}$ rate of the convergence. Finally, we illustrate the theoretical results by numerical simulations.
\end{abstract}

Keywords: thermal effects modelling; stochastic dynamical systems; convergence rate; ferromagnetism.

AMS Classification: 60F15, 60F25, 60Z05, 93E15.

\section{Introduction}

The use of stochastic modelling for ferromagnetic particles goes back to the seminal paper by Brown (1963) on the physical aspects of the problem. This problematic has been extensively studied by physicists in several application domains. For example, we can cite a wide range works on the suspension of heated magnetic particles going from Ivanov et al. (2016) for the microscale approach to studies implying thermal effects in larger magnetic structures such as those used in magnetic recording, see for instance Tserkovnyak et al. (2005). In this work, we focus on a single ferromagnetic mono-domain particle submitted to an external field, whose behaviour is usually modelled by the following deterministic dynamical system:

$$
\frac{d \mu}{d t}=-\mu \wedge b-\alpha \mu \wedge(\mu \wedge b), \quad \mu_{0} \in \mathcal{S}\left(\mathbb{R}^{3}\right)
$$

where $b \in \mathbb{R}^{3}$ is the external magnetic field, $\alpha \in \mathbb{R}_{+}$and $\mathcal{S}\left(\mathbb{R}^{3}\right)$ classically denotes the unit sphere in $\mathbb{R}^{3}$. It is clear that $\left|\mu_{t}\right|=1$ for all $t \geq 0$. We introduce the antisymmetric operator

*stephane.labbe@imag.fr

${ }^{\dagger}$ jerome.lelong@imag.fr 
$L: \mathbb{R}^{3} \longmapsto \mathcal{M}_{3 \times 3}$ associated to the cross product in $\mathbb{R}^{3}$

$$
L(x)=\left(\begin{array}{ccc}
0 & -x^{3} & x^{2} \\
x^{3} & 0 & -x^{1} \\
-x^{2} & x^{1} & 0
\end{array}\right)
$$

Let $A: \mathbb{R}^{3} \longrightarrow \mathcal{M}_{3 \times 3}$ be the operator defined by

$$
A(x)=\alpha x^{*} x I-\alpha x x^{*}-L(x)
$$

where $I$ is the identity matrix in $\mathcal{M}_{3 \times 3}$. We can write (1) as

$$
\frac{d \mu}{d t}=A(\mu) b, \quad \mu_{0} \in \mathcal{S}\left(\mathbb{R}^{3}\right) .
$$

This paper aims at introducing stochastic perturbations in order to model thermal effects. We already published an article Etoré et al. (2014) on the same subject but using a different modelling of the stochastic perturbation; in the present work, we use the Stratonovich integral to design an alternative model to the rescaled Itô approach studied in Etoré et al. (2014). First, we recall the model studied in the previous work along with the main results we obtained. Second, we explore two different approaches to model the effects of a heat pulse on the behaviour of a ferromagnet: we can either pull the Itô equation back onto the sphere with a suitable projection or we can interpret the stochastic model in the Stratonovich sense. We will see that both approaches actually coincide when considering the Euclidean projection. Then, we focus on the mathematical study of the Stratonovich approach and establish its long time convergence. Finally, we present some numerical results illustrating the theoretical results developed previously.

\section{Modelling issues}

Let $\left(\Omega, \mathcal{A}, \mathbb{F}=\left(\mathcal{F}_{t}\right)_{t \geq 0}, \mathbb{P}\right)$ be a filtered probability space. We consider a standard $\mathbb{F}$-Brownian motion $W$ with values in $\mathbb{R}^{3}$. Thermal effects can be embedded in the deterministic system (2) by adding a stochastic perturbation to the external field $b$, which naturally leads to the following stochastic system

$$
d \mu_{t}=A\left(\mu_{t}\right) b d t+\varepsilon A\left(\mu_{t}\right) d W_{t} .
$$

If this SDE is interpreted in the Itô sense, the system is not physically consistent as it does not preserve $\left|\mu_{t}\right|$ whereas this is a physical invariant of (2), see Brown (1962). In this section, we investigate several ways of modifying the stochastic model to ensure its consistency with the physical model. First, we recall the rescaled approach developed in Etoré et al. (2014). Second, we interpret the model (3) in the Stratonovich sense and study its long time behaviour.

\subsection{The Itô approach}

We recall the model developed in Etoré et al. (2014) along with some convergence results

$$
\begin{cases}d Y_{t} & =A\left(\mu_{t}\right) b d t+\varepsilon A\left(\mu_{t}\right) d W_{t} \\ \mu_{t} & =\frac{Y_{t}}{\left|Y_{t}\right|} \\ Y_{0} & =y \in \mathcal{S}\left(\mathbb{R}^{3}\right) .\end{cases}
$$


The process $\left(\mu_{t}\right)_{t}$ converges to $b$ when $t$ goes to infinity with the rate $\sqrt{t}$ : let $h(t)=\left|Y_{t}\right|=$ $\sqrt{2 \varepsilon^{2}\left(\alpha^{2}+1\right) t+1}$, which is deterministic for this model. The process $\mu$ solves the following $\mathrm{SDE}$

$$
d \mu_{t}=\left(-\frac{h^{\prime}(t)}{h(t)} \mu_{t}+\frac{A\left(\mu_{t}\right)}{h(t)} b\right) d t+\frac{\varepsilon}{h(t)} A\left(\mu_{t}\right) d W_{t},
$$

from which it clearly appears that the noise added to the model vanishes at the rate $\varepsilon h(t)^{-1}$. We recall the main result from Etoré et al. (2014) concerning the convergence of $\mu$ when $\alpha>0$.

Theorem 1. Assume $\alpha>0$. Then,

$$
\lim _{t \rightarrow \infty} \mu_{t}=b /|b| \text { a.s. } \quad \text { and } \quad \lim _{t \rightarrow \infty} \mathbb{E}\left[h(t)\left(|b|-\mu_{t} \cdot b\right)\right]=\frac{\varepsilon^{2}\left(\alpha^{2}+1\right)}{2 \alpha} .
$$

If instead of rescaling, we simply move the dynamics back on to the sphere with an extra term $K$, we get the following model

$$
d \mu_{t}=A\left(\mu_{t}\right) b d t+\varepsilon A\left(\mu_{t}\right) d W_{t}+K_{t} d t
$$

Using that $A(x) x=0$, we deduce that $d\left|\mu_{t}\right|^{2}=2 \mu_{t} \cdot K_{t} d t+\operatorname{tr}\left(d\langle\mu\rangle_{t}\right)$. An easy computation shows that $\operatorname{tr}\left(d\langle\mu\rangle_{t}\right)=2 \varepsilon^{2}\left(\alpha^{2}\left|\mu_{t}\right|^{2}+1\right)\left|\mu_{t}\right|^{2} d t$. Then, the condition $\left|\mu_{t}\right|=1$ imposes to choose $K_{t}=-\varepsilon^{2}\left(\alpha^{2}\left|\mu_{t}\right|^{2}+1\right) \mu_{t}+K_{t}^{\perp}$, where $K_{t}^{\perp}$ is orthogonal to $\mu_{t}$ for all $t$. The final term $K_{t}$ can be thought of as pulling the process $\mu$ back onto the sphere. The minimum norm pull is obtained by choosing $K^{\perp}=0$, which leads to

$$
d \mu_{t}=A\left(\mu_{t}\right) b d t+\varepsilon A\left(\mu_{t}\right) d W_{t}-\varepsilon^{2}\left(\alpha^{2}\left|\mu_{t}\right|^{2}+1\right) \mu_{t} d t
$$

and it simplifies into

$$
d \mu_{t}=A\left(\mu_{t}\right) b d t+\varepsilon A\left(\mu_{t}\right) d W_{t}-\varepsilon^{2}\left(\alpha^{2}+1\right) \mu_{t} d t
$$

as $\left|\mu_{t}\right|^{2}=1$. This equation will show up later as the Itô form of the Stratonovich stochastic model. The idea of taking for the $K_{t}$ the Euclidean projection can be used to define the spherical Brownian motion, see Brillinger (1997) for a survey of possible approaches to define the spherical Brownian motion.

\subsection{The Stratonovich approach}

Instead of trying to modify the Itô SDE (3) to satisfy the physical constraint $\left|\mu_{t}\right|=1$, it is possible to interpret the stochastic term $\varepsilon A\left(\mu_{t}\right) d W_{t}$ in the Stratonovich sense.

Let $\partial$ denote the Stratonovich differential operator. Let $\left(\bar{\mu}_{t}\right)_{t}$ denote the stochastic system with a Stratonovich perturbation. We assume that the magnitude of the stochastic perturbation is given by a deterministic positive function $\left(\varepsilon_{t}\right)_{t}$. In this section, we consider the stochastic model defined by the following Stratonovich SDE

$$
\partial \bar{\mu}_{t}=A\left(\bar{\mu}_{t}\right) b \partial t+\varepsilon_{t} A\left(\bar{\mu}_{t}\right) \partial W_{t} .
$$


If we compute $\partial\left|\bar{\mu}_{t}\right|^{2}=2 \bar{\mu}_{t} \cdot \partial \bar{\mu}_{t}$ using Equation (5), we immediately notice that $\partial\left|\bar{\mu}_{t}\right|^{2}=0$. Now, we turn this Stratonovich SDE into an Itô SDE (see (Rogers and Williams, 2000, V.30))

$$
d \bar{\mu}_{t}=A\left(\bar{\mu}_{t}\right) b d t+\varepsilon_{t} A\left(\bar{\mu}_{t}\right) d W_{t}+\frac{1}{2} \varepsilon_{t}^{2} \sum_{q=1}^{3} \sum_{j=1}^{3}\left(A_{j q} D_{j}\left(A_{i q}\right)\right)\left(\bar{\mu}_{t}\right),
$$

where $D_{j}$ denotes the partial derivative with respect to the $j-t h$ component.

$$
\sum_{q=1}^{3} \sum_{j=1}^{3}\left(A_{j q} D_{j}\left(A_{\cdot q}\right)\right)(x)=\sum_{j=1}^{3}\left(D_{j} A\right) A_{\cdot j}^{*}(x) .
$$

Let us compute $D_{j} A$ by using that $D_{j}(x)=\boldsymbol{e}_{j}$, where $\boldsymbol{e}_{j}$ is the $j-t h$ vector of the canonical basis.

$$
\begin{gathered}
D_{j} A(x)=\alpha\left(D_{j} x^{*} x+x^{*} D_{j} x\right) I-\alpha\left(D_{j} x x^{*}+x D_{j} x^{*}\right)-L\left(D_{j}(x)\right) \\
=\alpha\left(2 x^{*} \boldsymbol{e}_{j} I-\boldsymbol{e}_{j} x^{*}-x \boldsymbol{e}_{j}^{*}\right)-L\left(\boldsymbol{e}_{j}\right) . \\
\sum_{j=1}^{3}\left(D_{j} A\right) A_{\cdot j}^{*}(x)=\sum_{j=1}^{3}\left(-\alpha\left(-2 x^{*} \boldsymbol{e}_{j} I+\boldsymbol{e}_{j} x^{*}+x \boldsymbol{e}_{j}^{*}\right)-L\left(\boldsymbol{e}_{j}\right)\right)\left(\alpha|x|^{2} \boldsymbol{e}_{j}-\alpha x x_{j}-L\left(\boldsymbol{e}_{j}\right) x\right) \\
=\sum_{j=1}^{3} \alpha^{2}\left(2|x|^{2} x_{j} \boldsymbol{e}_{j}-2 x_{j}^{2} x-|x|^{2} \boldsymbol{e}_{j} x^{*} \boldsymbol{e}_{j}+\boldsymbol{e}_{j} x^{*} x x_{j}+-|x|^{2} x \boldsymbol{e}_{j}^{*} \boldsymbol{e}_{j}+x \boldsymbol{e}_{j}^{*} x x_{j}\right) \\
\quad+L\left(\boldsymbol{e}_{j}\right) L\left(\boldsymbol{e}_{j}\right) x .
\end{gathered}
$$

All the other terms vanish by using the linearity of the operator $L$. Hence,

$$
\sum_{j=1}^{3}\left(D_{j} A\right) A_{\cdot j}^{*}(x)=-2 \alpha^{2}|x|^{2}+\sum_{j=1}^{3}\left(\boldsymbol{e}_{j} \cdot x\right) \boldsymbol{e}_{j}-\left(\boldsymbol{e}_{j} \cdot \boldsymbol{e}_{j}\right) x=-2\left(\alpha^{2}|x|^{2}+1\right) x .
$$

Hence, $\left(\bar{\mu}_{t}\right)_{t}$ solves the following Itô SDE

$$
d \bar{\mu}_{t}=\left(A\left(\bar{\mu}_{t}\right) b-\varepsilon_{t}^{2}\left(\alpha^{2}\left|\bar{\mu}_{t}\right|^{2}+1\right) \bar{\mu}_{t}\right) d t+\varepsilon_{t} A\left(\bar{\mu}_{t}\right) d W_{t} .
$$

As by construction $\left|\bar{\mu}_{t}\right|^{2}=1$, the process $\left(\bar{\mu}_{t}\right)_{t}$ also solves

$$
d \bar{\mu}_{t}=\left(A\left(\bar{\mu}_{t}\right) b-\varepsilon_{t}^{2}\left(\alpha^{2}+1\right) \bar{\mu}_{t}\right) d t+\varepsilon_{t} A\left(\bar{\mu}_{t}\right) d W_{t} .
$$

This equation is similar to (4), which was obtained by pulling the Itô process back to the sphere. This similarity of the two equations actually advocates to interpret the noise in the Stratonovich sense as it naturally preserves the norm of $\mu$. However, it is easy to check that

$$
\left(d\left(\bar{\mu}_{t} \cdot b\right)\right)_{\bar{\mu}_{t}=b /|b|}=-\varepsilon_{t}^{2}\left(\alpha^{2}+1\right)|b| d t .
$$

This implies that $b$ cannot be an equilibrium point of the stochastic system $\left(\bar{\mu}_{t}\right)_{t}$ when $\varepsilon_{t}=$ $\varepsilon>0$ for all $t$. Moreover, for the constant magnitude case, it was proved in Etoré et al. (2014) that $\bar{\mu}_{t}$ could not converge to $b$. Actually, (6) very much looks like a continuous time stochastic approximation and it is known from Benaïm and Hirsch (1999); Fort and Pages (1999) that a long time stationary measure can only be obtained when letting $\varepsilon$ go to zero as well. Hence, it is natural to consider in the following the decreasing noise framework, ie. when $\varepsilon_{t}$ is a decreasing positive function. 


\section{The Stratonovich model with decreasing noise}

In this section, we assume that $\left(\varepsilon_{t}\right)_{t}$ is a decreasing function satisfying $\varepsilon_{t}>0$ for all $t \geq 0$ and $\lim _{t \rightarrow \infty} \varepsilon_{t}=0$.

$$
d \bar{\mu}_{t}=-\left(A\left(\bar{\mu}_{t}\right) b+\varepsilon_{t}^{2}\left(\alpha^{2}+1\right) \bar{\mu}_{t}\right) d t+\varepsilon_{t} A\left(\bar{\mu}_{t}\right) d W_{t},
$$

where the operator $A$ simplifies into $A(x)=\alpha I-\alpha x x^{*}-L(x)$ for $x \in \mathcal{S}\left(\mathbb{R}^{3}\right)$. Then, we deduce that

$$
\begin{aligned}
d\left(\bar{\mu}_{t} \cdot b\right)= & -\left\{\alpha\left(\left(\bar{\mu}_{t} \cdot b\right)^{2}-|b|^{2}\right)+\varepsilon_{t}^{2}\left(\alpha^{2}+1\right) \bar{\mu}_{t} \cdot b\right\} d t \\
& -\varepsilon_{t}\left(-L\left(\bar{\mu}_{t}\right) b+\alpha\left(\left(\bar{\mu}_{t} \cdot b\right) \bar{\mu}_{t}-b\right)\right) \cdot d W_{t} .
\end{aligned}
$$

As $\bar{\mu}$ is bounded, the stochastic integral in the above equation is a martingale with expectation zero. For future computations, it will be useful to know the quadratic variation of the process $\left(\bar{\mu}_{t} \cdot b\right)_{t}$ :

$$
d<\bar{\mu} \cdot b>_{t}=\varepsilon_{t}^{2}\left|-L\left(\bar{\mu}_{t}\right) b+\alpha\left(\left(\bar{\mu}_{t} \cdot b\right) \bar{\mu}_{t}-b\right)\right|^{2} d t=\varepsilon_{t}^{2}\left(\alpha^{2}+1\right)\left(|b|^{2}-\left(\bar{\mu}_{t} \cdot b\right)^{2}\right) d t .
$$

\subsection{The case $\alpha>0$}

Proposition 2. Assume that one of the following conditions holds

(i) $\int_{0}^{\infty} \varepsilon_{t}^{2} d t=\infty$ and $\int_{0}^{\infty} \varepsilon_{t}^{4} d t<\infty$.

(ii) $\int_{0}^{\infty} \varepsilon_{t}^{2} d t<\infty$.

Then, $\bar{\mu}_{t} \underset{t \rightarrow \infty}{\longrightarrow} \frac{b}{|b|}$ a.s.

The proof of this result heavily relies on a bespoke version of the ODE method, which aims at relating the behaviour of the SDE with the one of the ODE obtained by an averaging process. A general theory for the ODE method was developed in different frameworks by Benveniste et al. (1990); Benaim (1996); Kushner and Yin (2003). For more results on the stability of SDE, we refer to Khasminskii (2011).

Proof. As $\left|\bar{\mu}_{t}\right|=1$, the result is equivalent to $\bar{\mu}_{t} \cdot b \rightarrow|b|$ a.s.

Step 1. We aim at proving that the martingale part of $\left(\bar{\mu}_{t} \cdot b\right)_{t}$ (see (7)) converges a.s. Consider

$$
M_{t}=\int_{0}^{t} \varepsilon_{s}\left(-L\left(\bar{\mu}_{s}\right) b+\alpha\left(\left(\bar{\mu}_{s} \cdot b\right) \bar{\mu}_{s}-b\right)\right) \cdot d W_{s} .
$$

It is sufficient to prove that $\sup _{t} \mathbb{E}\left[M_{t}^{2}\right]<\infty$ to ensure the a.s. convergence of $M_{t}$.

$$
\mathbb{E}\left[M_{t}^{2}\right]=\int_{0}^{t} \varepsilon_{s}^{2}\left(\alpha^{2}+1\right) \mathbb{E}\left[|b|^{2}-\left(\bar{\mu}_{s} \cdot b\right)^{2}\right] d s .
$$

Case (ii). It is obvious that $\sup _{t} \mathbb{E}\left[M_{t}^{2}\right]<\infty$.

Case (i). From (7), we can deduce that

$$
\begin{array}{r}
\mathbb{E}\left[\bar{\mu}_{t} \cdot b\right]^{\prime}=\alpha \mathbb{E}\left[|b|^{2}-\left(\bar{\mu}_{t} \cdot b\right)^{2}\right]-\varepsilon_{t}^{2}\left(\alpha^{2}+1\right) \mathbb{E}\left[\bar{\mu}_{t} \cdot b\right] \\
\left(\mathbb{E}\left[\bar{\mu}_{t} \cdot b\right] \mathrm{e}^{\int_{0}^{t}\left(\alpha^{2}+1\right) \varepsilon_{s}^{2} d s}\right)^{\prime}=\alpha \mathbb{E}\left[|b|^{2}-\left(\bar{\mu}_{t} \cdot b\right)^{2}\right] \mathrm{e}^{\int_{0}^{t}\left(\alpha^{2}+1\right) \varepsilon_{s}^{2} d s} .
\end{array}
$$


Hence, as $\bar{\mu}_{t} \cdot b$ is bounded, there exists $\kappa>0$ such that for all $t \geq 0$

$$
\int_{0}^{t} \mathbb{E}\left[|b|^{2}-\left(\bar{\mu}_{s} \cdot b\right)^{2}\right] \mathrm{e}^{\int_{0}^{s}\left(\alpha^{2}+1\right) \varepsilon_{u}^{2} d u} d s \leq \kappa \mathrm{e}^{\int_{0}^{t}\left(\alpha^{2}+1\right) \varepsilon_{s}^{2} d s} .
$$

Combining (8) and (9), we obtain using an integration by parts formula that

$$
\begin{aligned}
\mathbb{E}\left[M_{t}^{2}\right] & =\int_{0}^{t} \varepsilon_{s}^{2}\left(\alpha^{2}+1\right) \mathrm{e}^{-\int_{0}^{s}\left(\alpha^{2}+1\right) \varepsilon_{u}^{2} d u} \mathbb{E}\left[|b|^{2}-\left(\bar{\mu}_{s} \cdot b\right)^{2}\right] \mathrm{e}^{\int_{0}^{s}\left(\alpha^{2}+1\right) \varepsilon_{u}^{2} d u} d s \\
& \leq \kappa \varepsilon_{t}^{2}\left(\alpha^{2}+1\right)+\kappa \int_{0}^{t}\left(\varepsilon_{s}^{2}\left(\alpha^{2}+1\right)\right)^{2}-2 \varepsilon_{s}^{\prime} \varepsilon_{s}\left(\alpha^{2}+1\right) d s \\
& \leq \kappa \varepsilon_{0}^{2}\left(\alpha^{2}+1\right)+\kappa \int_{0}^{t}\left(\varepsilon_{s}^{2}\left(\alpha^{2}+1\right)\right)^{2} d s .
\end{aligned}
$$

Considering the Assumption (i) satisfied by $\left(\varepsilon_{t}\right)_{t}, \sup _{t} \mathbb{E}\left[M_{t}^{2}\right]<\infty$.

In the end, when either (ii) or (ii) holds, the boundedness of $\mathbb{E}\left[M_{t}^{2}\right]$ ensures the a.s. convergence of the martingale $M$ to some square integrable random variable $M_{\infty}$ satisfying $M_{t}=\mathbb{E}\left[M_{\infty} \mid \mathcal{F}_{t}\right]$.

Step 2. Define $X_{t}=\bar{\mu}_{t} \cdot b-\left(M_{\infty}-M_{t}\right)$. The process $X$ solves the classical differential equation

$$
d X_{t}=-\left\{\alpha\left(\left(\bar{\mu}_{t} \cdot b\right)^{2}-|b|^{2}\right)+\varepsilon_{t}^{2}\left(\alpha^{2}+1\right) \bar{\mu}_{t} \cdot b\right\} d t .
$$

Let $\eta>0$, there exists $T>0$ s.t. for all $t \geq T, \varepsilon_{t}^{2}\left(\alpha^{2}+1\right)|b| \leq \eta$ and $\left|\bar{\mu}_{t} \cdot b-X_{t}\right| \leq \eta$.

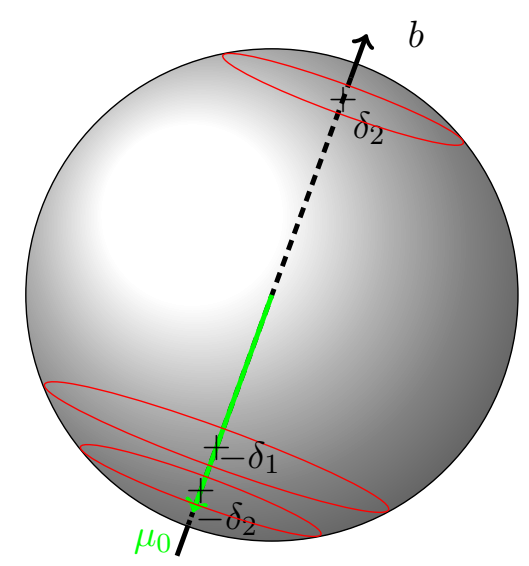

Figure 1: We consider three zones on the sphere $\left\{\bar{\mu} \cdot b \leq-\delta_{1}\right\},\left\{-\delta_{2} \leq \bar{\mu} \cdot b \leq \delta_{2}\right\}$ and $\left\{\bar{\mu} \cdot b \geq \delta_{2}\right\}$.

Let $0<\delta_{1}<\delta_{2}<|b|$. We can choose $\eta$ small enough such that $\delta_{1}<\delta_{2}-2 \eta$ and $\eta \leq$ $\frac{\alpha}{2}\left(|b|^{2}-\delta_{2}^{2}\right)$. Figure 1 defines three regions: the two pole caps and the region in between; depending on the position of $\bar{\mu}_{t} \cdot b$, we can bound from below the r.h.s of $(10)$ to deduce that 
for $t \geq s>T$.

$$
\begin{gathered}
X_{t}-X_{s} \geq \int_{s}^{t}\left(\alpha^{2}+1\right) \varepsilon_{u}^{2} \delta_{1} \mathbf{1}_{\left\{\bar{\mu}_{u} \cdot b \leq-\delta_{1}\right\}} d u+\int_{s}^{t} \frac{\alpha}{2}\left(|b|^{2}-\delta_{2}^{2}\right) \mathbf{1}_{\left\{-\delta_{2} \leq \bar{\mu}_{u} \cdot b \leq \delta_{2}\right\}} d u \\
\quad-\int_{s}^{t}|b| \varepsilon_{u}^{2}\left(\alpha^{2}+1\right) \mathbf{1}_{\left\{\bar{\mu}_{u} \cdot b>\delta_{2}\right\}} d u, \\
X_{t}-X_{s} \geq \int_{s}^{t}\left(\alpha^{2}+1\right) \varepsilon_{u}^{2} \delta_{1} \mathbf{1}_{\left\{X_{u} \leq-\delta_{1}-\eta\right\}} d u+\int_{s}^{t} \frac{\alpha}{2}\left(|b|^{2}-\delta_{2}^{2}\right) \mathbf{1}_{\left\{-\delta_{2}+\eta \leq X_{u} \leq \delta_{2}-\eta\right\}} d u \\
-\int_{s}^{t}|b| \varepsilon_{u}^{2}\left(\alpha^{2}+1\right) \mathbf{1}_{\left\{X_{u}>\delta_{2}+\eta\right\}} d u .
\end{gathered}
$$

Note that $X_{t}$ is increasing on the set $\left\{u: X_{u} \leq \delta_{2}-\eta\right\}$. We can choose $\delta_{2}$ sufficiently close to $|b|$ such that there exists $t_{1}$ for which $\int_{s}^{t_{1}}\left(\alpha^{2}+1\right) \varepsilon_{u}^{2} \delta_{1}=|b|-\left(\delta_{2}-\eta\right)$ - remember that $\eta$ can be chosen as small as necessary. Hence, for all $t \geq t_{1}, X_{t} \geq-\delta_{2}+\eta$. Therefore,

$$
X_{t}-X_{t_{1}} \geq \int_{t_{1}}^{t} \frac{\alpha}{2}\left(|b|^{2}-\delta_{2}^{2}\right) \mathbf{1}_{\left\{-\delta_{2}+\eta \leq X_{u} \leq \delta_{2}-\eta\right\}} d u-\int_{t_{1}}^{t}|b| \varepsilon_{u}^{2}\left(\alpha^{2}+1\right) \mathbf{1}_{\left\{X_{u} \geq \delta_{2}+\eta\right\}} d u .
$$

From the continuity of $X$, we deduce that there exists $t_{2} \geq t_{1}$ such that for all $t \geq t_{2}$, $X_{t} \geq \delta_{2}-\eta$, which implies that for all $t \geq t_{2}, \bar{\mu}_{t} \cdot b \geq \delta_{2}-2 \eta$. By choosing $\delta$ close to $|b|$ and $\eta$ close to 0 , we find that $\bar{\mu}_{t} \cdot b \rightarrow|b|$.

Proposition 3. Assume $\bar{\mu}_{t} \rightarrow b /|b|$ a.s. If $\left(\varepsilon_{t}\right)_{t}$ is of class $C^{1}$ and $\lim _{t \rightarrow \infty} \frac{\varepsilon_{t}^{\prime}}{\varepsilon_{t}}=0$, then, for all $p \in \mathbb{N}$,

$$
\lim _{t \rightarrow \infty} \mathbb{E}\left[\left|\frac{b}{|b|}-\bar{\mu}_{t}\right|^{2 p} \varepsilon_{t}^{-2 p}\right]=\left(\frac{\alpha^{2}+1}{\alpha|b|}\right)^{p} p ! .
$$

Note that as $\left|\bar{\mu}_{t}\right|=1,\left|\frac{b}{|b|}-\bar{\mu}_{t}\right|^{2}=\frac{2}{\mid b}\left(|b|-\bar{\mu}_{t} \cdot b\right)$. Hence, the proposition could equivalently write $\lim _{t \rightarrow \infty} \mathbb{E}\left[\left(|b|-\bar{\mu}_{t} \cdot b\right)^{p} \varepsilon_{t}^{-2 p}\right]=\left(\frac{\alpha^{2}+1}{2 \alpha}\right)^{p} p$ !. For $p=1$, this boils down to $\lim _{t \rightarrow \infty} \mathbb{E}[(|b|-$ $\left.\left.\bar{\mu}_{t} \cdot b\right) \varepsilon_{t}^{-2}\right]=\frac{\alpha^{2}+1}{2 \alpha}$. In the case of the rescaled Itô model, the convergence rate was given by $h(t)$, which actually monitors the magnitude of the noise. In the Stratonovich model, this role is played by $\varepsilon_{t}$. Hence, we would have expected a convergence rate of $\varepsilon_{t}^{-1}$ whereas we obtained a much faster one, namely the square of it $-\varepsilon_{t}^{-2}$. Although in both models, the magnetic moment converges to $b$, the rates governing the convergence significantly differ.

Proof. Step 1. Consider the process $Y^{p}$ defined by $Y_{t}^{p}=\left(|b|-\bar{\mu}_{t} \cdot b\right)^{p+1} \varepsilon_{t}^{-2 p}$. We aim at proving that, for all $p \in \mathbb{N}, \mathbb{E}\left[Y_{t}^{p}\right] \rightarrow 0$. For $p=0$, the result follows from the almost sure convergence of $\bar{\mu}_{t} \cdot b$ combined with the bounded convergence theorem. Now, we prove the statement by induction for $p \geq 1$. Assume the convergence holds true for $p-1$. Applying the 
Itô formula to $Y^{p}$ yields.

$$
\begin{aligned}
d Y_{t}^{p}= & -(p+1) \varepsilon_{t}^{-2 p}\left(|b|-\bar{\mu}_{t} \cdot b\right)^{p} d\left(\bar{\mu}_{t} \cdot b\right)-2 p \varepsilon_{t}^{\prime} \varepsilon_{t}^{-1} Y_{t}^{p} d t+\frac{p(p+1)}{2}\left(|b|-\bar{\mu}_{t} \cdot b\right)^{p-1} \varepsilon_{t}^{-2 p} d\langle\bar{\mu} \cdot b\rangle_{t} \\
= & (p+1) \varepsilon_{t}^{-2 p}\left(|b|-\bar{\mu}_{t} \cdot b\right)^{p}\left\{\alpha\left(\left(\bar{\mu}_{t} \cdot b\right)^{2}-|b|^{2}\right)+\varepsilon_{t}^{2}\left(\alpha^{2}+1\right) \bar{\mu}_{t} \cdot b\right\} d t-2 p \varepsilon_{t}^{\prime} \varepsilon_{t}^{-1} Y_{t}^{p} d t \\
& +\frac{p(p+1)}{2}\left(\alpha^{2}+1\right) Y_{t}^{p-1}\left(|b|+\bar{\mu}_{t} \cdot b\right) d t+\ldots d W_{t} \\
= & \left\{-(p+1) \alpha Y_{t}^{p}\left(\left(\bar{\mu}_{t} \cdot b\right)+|b|\right)-2 p \varepsilon_{t}^{\prime} \varepsilon_{t}^{-1} Y_{t}^{p}\right\} d t \\
& +(p+1)\left(\alpha^{2}+1\right) Y_{t}^{p-1}\left(\frac{p+2}{2} \bar{\mu}_{t} \cdot b+\frac{p}{2}|b|\right) d t+\ldots d W_{t} .
\end{aligned}
$$

Introduce the function $F(t)=2 p \log \varepsilon_{t}+(p+1) \alpha|b| t$. As $\lim _{t \rightarrow \infty} \varepsilon_{t}^{\prime} / \varepsilon_{t}=0, \log \varepsilon_{t}=o(t)$ when $t \rightarrow \infty$ and therefore $F(t) \sim(p+1) \alpha|b| t$. Then, we integrate the above differential equation to obtain

$$
\begin{aligned}
\mathbb{E}\left[Y_{t}^{p}\right]-\mathbb{E}\left[Y_{0}^{p}\right] \mathrm{e}^{F(0)-F(t)} & =\mathrm{e}^{-F(t)} \int_{0}^{t} \mathrm{e}^{F(s)}(p+1)\left(\alpha^{2}+1\right) \mathbb{E}\left[Y_{s}^{p-1}\left(\frac{p+2}{2} \bar{\mu}_{s} \cdot b+\frac{p}{2}|b|\right]\right) d s \\
& -\mathrm{e}^{-F(t)} \int_{0}^{t} \mathrm{e}^{F(s)}(p+1) \alpha \mathbb{E}\left[Y_{s}^{p}\left(\bar{\mu}_{s} \cdot b\right)\right] d s .
\end{aligned}
$$

The result for $p-1$ combined with Lemma 7 yields that the first term on the r.h.s tends to 0 and we are left with

$$
\limsup _{t} \mathbb{E}\left[Y_{t}^{p}\right]=-\liminf \mathrm{e}^{-F(t)} \int_{0}^{t} \mathrm{e}^{F(s)}(p+1) \alpha \mathbb{E}\left[Y_{s}^{p}\left(\bar{\mu}_{s} \cdot b\right)\right] d s .
$$

From Lemma 8, we deduce

$$
\underset{t}{\limsup } \mathbb{E}\left[Y_{t}^{p}\right] \leq-\liminf \frac{1}{|b|} \mathbb{E}\left[Y_{t}^{p}\left(\bar{\mu}_{t} \cdot b\right)\right] \leq 0
$$

where the last inequality comes from Fatou's lemma. Then, we conclude that

$$
\text { for all } p \geq 0, \lim _{t} \mathbb{E}\left[Y_{t}^{p}\right]=0 \text {. }
$$

Step 2. Define $Z_{t}^{p}=\left(|b|-\bar{\mu}_{t} \cdot b\right)^{p} \varepsilon_{t}^{-2 p}$. We aim at proving that $\lim _{t \rightarrow \infty} \mathbb{E}\left[Z_{t}^{p}\right]=\left(\frac{\alpha^{2}+1}{2 \alpha}\right)^{p} p$ ! by induction. The result is obvious for $p=0$ using the a.s. convergence of $\bar{\mu}_{t}$ to $b$. Assume $p \geq 1$ in the following.

$$
\begin{aligned}
d Z_{t}^{p}= & -p \varepsilon_{t}^{-2 p}\left(|b|-\bar{\mu}_{t} \cdot b\right)^{p-1} d\left(\bar{\mu}_{t} \cdot b\right)-2 p \varepsilon_{t}^{\prime} \varepsilon_{t}^{-1} Z_{t}^{p} d t+\frac{p(p-1)}{2}\left(|b|-\bar{\mu}_{t} \cdot b\right)^{p-2} \varepsilon_{t}^{-2 p} d\langle\bar{\mu} \cdot b\rangle_{t} \\
= & p \varepsilon_{t}^{-2 p}\left(|b|-\bar{\mu}_{t} \cdot b\right)^{p-1}\left\{\alpha\left(\left(\bar{\mu}_{t} \cdot b\right)^{2}-|b|^{2}\right)+\varepsilon_{t}^{2}\left(\alpha^{2}+1\right) \bar{\mu}_{t} \cdot b\right\} d t-2 p \varepsilon_{t}^{\prime} \varepsilon_{t}^{-1} Z_{t}^{p} d t \\
& +\frac{p(p-1)}{2}\left(\alpha^{2}+1\right) Z_{t}^{p-1}\left(|b|+\bar{\mu}_{t} \cdot b\right) d t+\ldots d W_{t} \\
= & \left\{-p \alpha Z_{t}^{p}\left(\left(\bar{\mu}_{t} \cdot b\right)+|b|\right)-2 p \varepsilon_{t}^{\prime} \varepsilon_{t}^{-1} Z_{t}^{p}+p\left(\alpha^{2}+1\right) Z_{t}^{p-1}\left(\bar{\mu}_{t} \cdot b+\frac{p-1}{2}\left(|b|+\bar{\mu}_{t} \cdot b\right)\right)\right\} d t+\ldots d W_{t} .
\end{aligned}
$$


If we group terms and take expectation, we obtain

$$
\begin{aligned}
\mathbb{E}\left[Z_{t}^{p}\right]^{\prime}= & \left(-2 p \varepsilon_{t}^{\prime} \varepsilon_{t}^{-1}-2 p \alpha|b|\right) \mathbb{E}\left[Z_{t}^{p}\right]+p \alpha \mathbb{E}\left[Z_{t}^{p}\left(|b|-\bar{\mu}_{t} \cdot b\right)\right] \\
& +\left(\alpha^{2}+1\right) p \mathbb{E}\left[Z_{t}^{p-1}\left(\frac{p+1}{2}\left(\bar{\mu}_{t} \cdot b-|b|\right)+p|b|\right)\right] .
\end{aligned}
$$

Note that $Z_{t}^{p}\left(|b|-\bar{\mu}_{t} \cdot b\right)=Y_{t}^{p}$. Then, using the function $G(t)=2 p \log \left(\varepsilon_{t}\right)+2 p \alpha|b| t$, we can write

$$
\left(\mathbb{E}\left[Z_{t}^{p}\right] \mathrm{e}^{G(t)}\right)^{\prime}=\left\{p \alpha \mathbb{E}\left[Y_{t}^{p}\right]+\left(\alpha^{2}+1\right) p \mathbb{E}\left[-\frac{p+1}{2} Y_{t}^{p-1}+p Z_{t}^{p-1}|b|\right]\right\} \mathrm{e}^{G(t)}
$$

Integrating this differential equation leads to

$$
\begin{aligned}
\mathbb{E}\left[Z_{t}^{p}\right]- & \mathbb{E}\left[Z_{0}^{p}\right] \mathrm{e}^{G(0)-G(t)}=\mathrm{e}^{-G(t)} \int_{0}^{t}\left(\alpha^{2}+1\right) p^{2}|b| \mathbb{E}\left[Z_{s}^{p-1}\right] \mathrm{e}^{G(s)} d s \\
& +\mathrm{e}^{-G(t)} \int_{0}^{t} p\left\{\alpha \mathbb{E}\left[Y_{s}^{p}\right]-\left(\alpha^{2}+1\right) \frac{p(p+1)}{2} \mathbb{E}\left[Y_{s}^{p-1}\right]\right\} \mathrm{e}^{G(s)} d s .
\end{aligned}
$$

From the first step of the proof of (11), the second term on the r.h.s of 12 tends to 0 when $t$ goes to infinity.

Assume the result holds true for $p-1$, ie $\lim _{t} \mathbb{E}\left[Z_{t}^{p-1}\right]=\left(\frac{a^{2}+1}{2 \alpha}\right)^{p-1}(p-1)$ !, then

$$
\lim _{t \rightarrow \infty} \mathbb{E}\left[Z_{t}^{p}\right]=\lim _{t \rightarrow \infty} \mathrm{e}^{-G(t)} \int_{0}^{t}\left(\alpha^{2}+1\right) p^{2}|b| \mathbb{E}\left[Z_{s}^{p-1}\right] \mathrm{e}^{G(s)} d s
$$

With the help of Lemma 7, we easily conclude that

$$
\lim _{t} \mathbb{E}\left[Z_{t}^{p}\right]=\left(\frac{a^{2}+1}{2 \alpha}\right)^{p} p !
$$

Proposition 4. Assume that

- there exists $\gamma>0$ such that $\int_{0}^{\infty} \varepsilon_{t}^{\gamma} d t<\infty$;

- the function $\left(\varepsilon_{t}\right)_{t}$ is $C^{1}$, decreasing for large enough $t$ and satisfies $\lim _{t \rightarrow \infty} \frac{\varepsilon_{t}^{\prime}}{\varepsilon_{t}}=0$.

Then,

$$
\text { for all } \eta>0,\left|\frac{b}{|b|}-\bar{\mu}_{t}\right|^{2} \varepsilon_{t}^{-2+\eta} \rightarrow 0 \text { a.s. }
$$

Proof. Step 1. As $\left(\varepsilon_{t}\right)_{t}$ is decreasing, then for all $t \geq s$ and all $\beta \geq 0$, we have $\varepsilon_{t}^{\gamma+\beta} \leq \varepsilon_{t}^{\gamma} \varepsilon_{s}^{\beta}$. Hence, for all $\beta>0, \int_{0}^{\infty} \varepsilon_{t}^{\gamma+\beta} d t<\infty$.

First, we only prove the result for integer values of $t$. From Proposition 3 .

$$
\mathbb{E}\left[\left|\frac{b}{|b|}-\bar{\mu}_{t}\right|^{2 p} \varepsilon_{t}^{-2 p+\eta p}\right] \geq C_{p} \varepsilon_{t}^{\eta p}
$$


for all $p \geq 1$ where $C_{p}>0$ is independent of $t$.

$$
\sum_{t=0}^{\infty} \mathbb{E}\left[\left|\frac{b}{|b|}-\bar{\mu}_{t}\right|^{2 p} \varepsilon_{t}^{-2 p+\eta p}\right] \leq \sum_{t=0}^{\infty} C_{p} \varepsilon_{t}^{\eta p} \leq C_{p} \int_{0}^{\infty} \varepsilon_{t}^{\eta p} d t .
$$

Choose $p$ such that $\eta p \geq \gamma$ and hence, $\int_{0}^{\infty} \varepsilon_{t}^{\eta p} d t<\infty$. Then, for such a $p$,

$$
\sum_{t=0}^{\infty} \mathbb{E}\left[\left|\frac{b}{|b|}-\bar{\mu}_{t}\right|^{2 p} \varepsilon_{t}^{-2 p+\eta p}\right]=\mathbb{E}\left[\sum_{t=0}^{\infty}\left|\frac{b}{|b|}-\bar{\mu}_{t}\right|^{2 p} \varepsilon_{t}^{-2 p+\eta p}\right]<\infty .
$$

Consequently, $\sum_{t=0}^{\infty}\left|\frac{b}{|b|}-\bar{\mu}_{t}\right|^{2 p} \varepsilon_{t}^{-2 p+\eta p}<\infty$ a.s. finite and therefore

$$
\lim _{t \in \mathbb{N}, t \rightarrow \infty}\left|\frac{b}{|b|}-\bar{\mu}_{t}\right|^{2} \varepsilon_{t}^{-2+\eta}=0 \text { a.s. }
$$

This standard reasoning relies on the Borel-Cantelli lemma, which cannot be applied readily for a family of continuous time events. Extending this result to $t \in \mathbb{R}_{+}$requires to monitor the behaviour in $L^{p}(\Omega)$ of $\left|\frac{b}{|b|}-\bar{\mu}_{t}\right|^{2} \varepsilon_{t}^{-2}$ for $t \in[n, n+1]$ for any $n \in \mathbb{N}$.

Step 2. We aim at proving that $\lim _{n \rightarrow \infty} \sup _{n \leq t \leq n+1}|| \frac{b}{|b|}-\left.\bar{\mu}_{t}\right|^{2} \varepsilon_{t}^{-2+\eta}-\left|\frac{b}{|b|}-\bar{\mu}_{n}\right|^{2} \varepsilon_{n}^{-2+\eta} \mid \rightarrow$ 0 a.s. Define $X_{t}=\frac{b}{|b|}-\bar{\mu}_{t}$. Let $n \in \mathbb{N}$ and $n \leq t \leq n+1$

$$
\begin{aligned}
\left.|| X_{t}\right|^{2} \varepsilon_{t}^{-2+\eta}-\left|X_{n}\right|^{2} \varepsilon_{n}^{-2+\eta} \mid & \leq\left. C|| X_{n}\right|^{2}\left(\varepsilon_{n}^{-2+\eta}-\varepsilon_{t}^{-2+\eta}\right)|+C|\left(\left|X_{n}\right|^{2}-\left|X_{t}\right|^{2}\right) \varepsilon_{t}^{-2+\eta} \mid \\
& \leq\left|X_{n}\right|^{2} \varepsilon_{n}^{-2+\eta}+\left|\left(\left|X_{n}\right|^{2}-\left|X_{t}\right|^{2}\right) \varepsilon_{t}^{-2+\eta}\right| \\
& \leq\left|X_{n}\right|^{2} \varepsilon_{n}^{-2+\eta}+\left|\left(\left|X_{n}\right|^{2}-\left|X_{t}\right|^{2}\right) \varepsilon_{n}^{-2+\eta}\right| .
\end{aligned}
$$

As we know from 13 that $\left|X_{n}\right|^{2} \varepsilon_{n}^{-2+\eta} \rightarrow 0$, it is sufficient to monitor $\left.\sup _{n \leq t \leq n+1}|| X_{n}\right|^{2}-\left|X_{t}\right|^{2} \mid \varepsilon_{n}^{-2+\eta}$. It is acutally enough to consider $\sup _{n \leq t \leq n+1}\left|X_{n}-X_{t}\right| \varepsilon_{n}^{-2+\eta}$ as $\left|X_{t}\right| \leq 2$.

Let $p>1$.

$$
\begin{aligned}
& \mathbb{E}\left[\sup _{n \leq t \leq n+1}\left|X_{t}-X_{n}\right|^{2 p}\right] \\
& \leq C \mathbb{E}\left[\sup _{n \leq t \leq n+1}\left|\int_{n}^{t} \alpha\left(|b|^{2}-\left(\bar{\mu}_{u} \cdot b\right)^{2}\right)-\varepsilon_{u}^{2}\left(\alpha^{2}+1\right) \bar{\mu}_{u} \cdot b d u\right|^{2 p}\right] \\
& \quad+C \mathbb{E}\left[\sup _{n \leq t \leq n+1}\left|\int_{n}^{t} \varepsilon_{u}\left(\bar{\mu}_{u} \wedge b+\alpha\left(b-\left(\bar{\mu}_{u} \cdot b\right) \bar{\mu}_{u}\right)\right) \cdot d W_{u}\right|^{2 p}\right] \\
& \leq C \mathbb{E}\left[\int_{n}^{n+1} \alpha^{2 p}\left(|b|^{2}-\left(\bar{\mu}_{u} \cdot b\right)^{2}\right)^{2 p}+\varepsilon_{u}^{4 p}\left(\alpha^{2}+1\right)^{2 p} d u\right] \\
& \quad+C \mathbb{E}\left[\left(\int_{n}^{n+1} \varepsilon_{u}^{2}\left(\alpha^{2}+1\right)\left(|b|^{2}-\left(\bar{\mu}_{t} \cdot b\right)^{2}\right) d u\right)^{p}\right]
\end{aligned}
$$


where we have used Burkholder-Davis-Gundi's inequality to treat the stochastic integral term. From Proposition $3, \mathbb{E}\left[\left(|b|^{2}-\left(\bar{\mu}_{u} \cdot b\right)^{2}\right)^{p}\right]=O\left(\varepsilon_{u}^{2 p}\right)$ and we can write

$$
\begin{aligned}
& \mathbb{E}\left[\sup _{n \leq t \leq n+1}\left|X_{t}-X_{n}\right|^{2 p}\right] \\
& \leq C \int_{n}^{n+1} \mathbb{E}\left[\left(|b|^{2}-\left(\bar{\mu}_{u} \cdot b\right)^{2}\right)^{2 p}\right]+\varepsilon_{u}^{4 p} d u+C \int_{n}^{n+1} \mathbb{E}\left[\varepsilon_{u}^{2 p}\left(|b|^{2}-\left(\bar{\mu}_{u} \cdot b\right)^{2}\right)^{p}\right] d u \\
& \leq C \int_{n}^{n+1} \varepsilon_{u}^{4 p} d u \leq C \varepsilon_{n}^{4 p} .
\end{aligned}
$$

Hence, we obtain

$$
\begin{aligned}
& \mathbb{E} {\left[\sup _{n \leq t \leq n+1}\left(\varepsilon_{n}^{-2+\eta}\left|X_{t}-X_{n}\right|\right)^{2 p}\right] \leq C \varepsilon_{n}^{p \eta} \leq C \int_{n-1}^{n} \varepsilon_{u}^{p \eta} d u } \\
& \sum_{n \geq 1} \mathbb{E}\left[\sup _{n \leq t \leq n+1}\left(\varepsilon_{n}^{-2+\eta}\left|X_{t}-X_{n}\right|\right)^{2 p}\right] \leq C \int_{0}^{\infty} \varepsilon_{u}^{p \eta} d u .
\end{aligned}
$$

For $p \eta \geq \gamma, \int_{0}^{\infty} \varepsilon_{u}^{p \eta} d u<\infty$ and Borel-Cantelli's lemma yields that

$$
\lim _{n \rightarrow \infty} \sup _{n \leq t \leq n+1} \varepsilon_{n}^{-2+\eta}\left|X_{t}-X_{n}\right|^{2}=0 .
$$

Then, we easily conclude that (13) holds for any real $t$ and not only integers.

\subsection{The case $\alpha=0$}

In this case, the process $\bar{\mu}$ solves the simplified equation

$$
d \bar{\mu}_{t}=\left(L(b)-\varepsilon_{t}^{2} I\right) \bar{\mu}_{t} d t-\varepsilon_{t} L\left(\bar{\mu}_{t}\right) d W_{t} .
$$

We can integrate this SDE as a classical ODE to obtain

$$
\begin{aligned}
& d\left(\mathrm{e}^{-L(b) t+\int_{0}^{t} \varepsilon_{u}^{2} d u} \bar{\mu}_{t}\right)=-\mathrm{e}^{-L(b) t+\int_{0}^{t} \varepsilon_{u}^{2} d u} \varepsilon_{t} L\left(\bar{\mu}_{t}\right) d W_{t} \\
& \bar{\mu}_{t}-\mathrm{e}^{L(b) t-\int_{0}^{t} \varepsilon_{u}^{2} d u} \bar{\mu}_{0}=-\mathrm{e}^{L(b) t-\int_{0}^{t} \varepsilon_{u}^{2} d u} \int_{0}^{t} \mathrm{e}^{-L(b) s+\int_{0}^{s} \varepsilon_{u}^{2} d u} \varepsilon_{s} L\left(\bar{\mu}_{s}\right) d W_{s} .
\end{aligned}
$$

Let us introduce the square integrable martingale $N$ defined by

$$
N_{t}=\int_{0}^{t} \mathrm{e}^{-L(b) s+\int_{0}^{s} \varepsilon_{u}^{2} d u} \varepsilon_{s} L\left(\bar{\mu}_{s}\right) d W_{s} .
$$

The long behaviour of $\left(\bar{\mu}_{t}\right)_{t}$ depends on the integrability of $\left(\varepsilon_{t}\right)_{t}$.

Proposition 5. If $\int_{0}^{\infty} \varepsilon_{u}^{2} d u=\infty, \mathbb{E}\left[\bar{\mu}_{t}\right] \rightarrow 0$ when $t \rightarrow \infty$.

Proof. From Eq. (15), we deduce that

$$
\left|\mathbb{E}\left[\bar{\mu}_{t}\right]\right| \leq \mathrm{e}^{-\int_{0}^{t} \varepsilon_{u}^{2} d u}\left\|\mathrm{e}^{L(b) t}\right\|\left|\bar{\mu}_{0}\right| \leq \mathrm{e}^{-\int_{0}^{t} \varepsilon_{u}^{2} d u}\left|\bar{\mu}_{0}\right| .
$$

The non integrability of $\left(\varepsilon_{t}^{2}\right)_{t}$ yields the result. 
Proposition 6. When $\int_{0}^{\infty} \varepsilon_{u}^{2} d u<\infty, N_{t}$ converges a.s. to some random $N_{\infty}$ and

$$
\lim _{t \rightarrow \infty} \bar{\mu}_{t}-\mathrm{e}^{L(b) t-\int_{0}^{\infty} \varepsilon_{u}^{2} d u}\left(\bar{\mu}_{0}-N_{\infty}\right)=0 \text { a.s }
$$

and moreover for all $p \in \mathbb{N}$, there exists $c_{p}$, such that $\mathbb{E}\left[\left|N_{t}\right|^{2 p}\right] \leq c_{p}\left(\mathrm{e}^{2 \int_{0}^{t} \varepsilon_{s}^{2} d s}-1\right)^{p}$ for all $t \geq 0$.

When $\int_{0}^{\infty} \varepsilon_{u}^{2} d u<\infty, \sup _{t} \mathbb{E}\left[\left|N_{t}\right|^{2}\right]<\infty$ and then $N$ converges a.s. to $N_{\infty}$ and $N_{t}=\mathbb{E}\left[N_{\infty} \mid \mathcal{F}_{t}\right]$. Hence, we clearly have $\mathbb{E}\left[N_{\infty}\right]=0$ and we obtain that $\lim _{t \rightarrow \infty} \mathbb{E}\left[\bar{\mu}_{t}\right]-\mathrm{e}^{L(b) t-\int_{0}^{t} \varepsilon_{u}^{2} d u} \bar{\mu}_{0}=0$ a.s. The term $\mathrm{e}^{L(b) t}$ makes $\bar{\mu}_{t}$ move on the ring with level $\mathrm{e}^{-\int_{0}^{\infty} \varepsilon_{u}^{2} d u}\left(\bar{\mu}_{0} \cdot b-N_{\infty} \cdot b\right)$.

Proof. From 15), we have

$$
\bar{\mu}_{t}-\mathrm{e}^{L(b) t-\int_{0}^{t} \varepsilon_{u}^{2} d u}\left(\bar{\mu}_{0}-N_{t}\right)=0
$$

Hence, it is sufficient to prove that $\mathrm{e}^{L(b) t-\int_{0}^{t} \varepsilon_{u}^{2} d u}\left(\bar{\mu}_{0}-N_{t}\right)-\mathrm{e}^{L(b) t-\int_{0}^{\infty} \varepsilon_{u}^{2} d u}\left(\bar{\mu}_{0}-N_{\infty}\right) \rightarrow 0$ a.s.

$$
\begin{aligned}
& \left|\mathrm{e}^{L(b) t}\left(\mathrm{e}^{-\int_{0}^{t} \varepsilon_{u}^{2} d u}\left(\bar{\mu}_{0}-N_{t}\right)-\mathrm{e}^{\int_{0}^{\infty} \varepsilon_{u}^{2} d u}\left(\bar{\mu}_{0}-N_{\infty}\right)\right)\right| \\
& \leq\left|-\mathrm{e}^{-\int_{t}^{\infty} \varepsilon_{u}^{2} d u} \bar{\mu}_{0}-\mathrm{e}^{-\int_{0}^{t} \varepsilon_{u}^{2} d u} N_{t}+\mathrm{e}^{\int_{0}^{\infty} \varepsilon_{u}^{2} d u} N_{\infty}\right| \\
& \leq\left|-\mathrm{e}^{-\int_{t}^{\infty} \varepsilon_{u}^{2} d u} \bar{\mu}_{0}\right|+\left|-\mathrm{e}^{-\int_{0}^{t} \varepsilon_{u}^{2} d u} N_{t}+\mathrm{e}^{\int_{0}^{\infty} \varepsilon_{u}^{2} d u} N_{\infty}\right| \rightarrow 0 .
\end{aligned}
$$

This last result yields the convergence announced in the Proposition.

Let $p \in \mathbb{N}^{*}$. Using Burkholder-Davis-Gundi's inequality (see Revuz and Yor (1999)), we have for $c_{p}=\left(\frac{p(p-1)}{2}\left(\frac{p}{p-1}\right)^{p}\right)^{p / 2}$

$$
\mathbb{E}\left[\left|N_{t}\right|^{2 p}\right] \leq c_{p} \mathbb{E}\left[\left|\langle N\rangle_{t}\right|^{p}\right] \leq c_{p} \mathbb{E}\left[\left(\int_{0}^{t} \varepsilon_{s}^{2} \mathrm{e}^{2 \int_{0}^{s} \varepsilon_{u}^{2} d u} d s\right)^{p}\right] \leq c_{p} 2^{-p}\left(\mathrm{e}^{2 \int_{t}^{\infty} \varepsilon_{u}^{2} d u}-1\right)^{p} .
$$

When $\varepsilon_{t}=\frac{\varepsilon}{(t+1)^{1 / 2+\eta}}$ where $\eta>0$ and $\varepsilon>0$, we can explicitly compute the upper-bound on $\mathbb{E}\left[\left|N_{t}\right|^{2 p}\right]$

$$
\left(\mathrm{e}^{2 \int_{0}^{t} \varepsilon_{s}^{2} d s}-1\right)^{p}=\left(\mathrm{e}^{\frac{\varepsilon^{2}}{\eta}\left(1-(t+1)^{-2 \eta}\right)}-1\right)^{p} \rightarrow\left(\mathrm{e}^{\frac{\varepsilon^{2}}{\eta}}-1\right)^{p}
$$

Usually, $\varepsilon^{2} \ll \eta$ and therefore $\left(\mathrm{e}^{\frac{\varepsilon^{2}}{\eta}}-1\right)^{p} \approx\left(\frac{\varepsilon^{2}}{\eta}\right)^{p}$. Hence, with a very high probability, $N_{\infty}$ remains tiny, and then for large $t, \bar{\mu}_{t}$ oscillates as $\mathrm{e}^{L(b) t-\int_{0}^{\infty} \varepsilon_{u}^{2} d u} \bar{\mu}_{0}$, which is non random.

\section{Numerical simulations}

In this section, we illustrate the theoretical results of Section 3 for different values of $\alpha$ and functions $\left(\varepsilon_{t}\right)_{t}$ with different decaying rates. To discretize the Stratonovich model (5), we would rather consider its Itô form given by (6), on which we use an Euler scheme with 
time step $\Delta t$. The Euler scheme has the advantage of being fully explicit and therefore can be easily implemented. We could have straightaway discretized the Stratonovich form (5), but the discretization of the Stratonovich integral must be performed using a semi-implicit scheme, which requires the use of a numerical solver at each iteration. In our case, the use of a semi-implicit scheme would have preserved the norm of the discretized process, which is not guarantied by an explicit scheme. However, using the Euler scheme on the Itô form did not raise any numerical difficulty.

Some of the graphs below have required to compute expectations, which were approximated using a Monte Carlo method with 500 samples. This may seem few samples but it proved to be enough as the quantities involved have little variance especially when focusing on the behaviour for large times.

\subsection{The case $\alpha>0$}

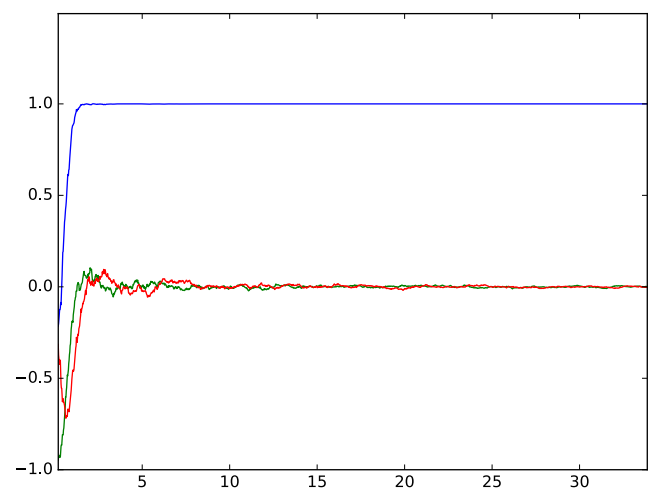

(a) A.s. convergence

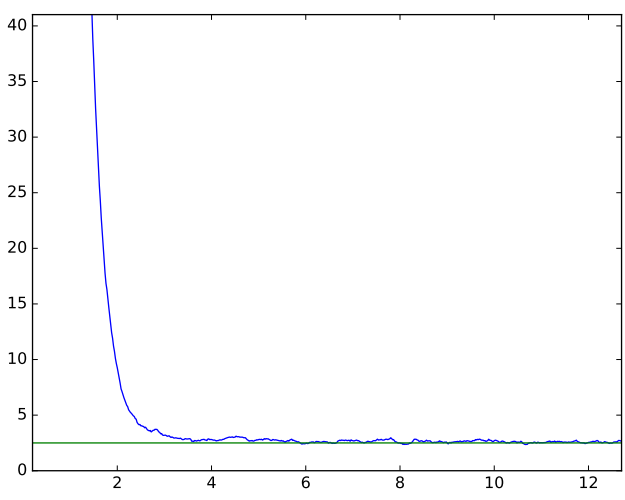

(b) Convergence rate in $L^{2}$ of $\left|\frac{b}{|b|}-\bar{\mu}_{t}\right| \varepsilon_{t}^{-1}$

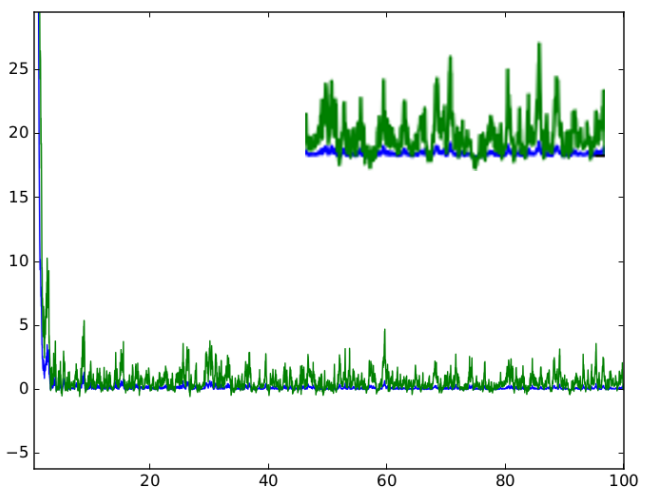

(c) Pathwise Convergence of $\left|\frac{b}{|b|}-\bar{\mu}_{t}\right| \varepsilon_{t}^{-1+\eta}$ for $\eta=$ 0.25 (blue curve) or $\eta=0.1$ (green curve)

Figure 2: Convergence of $\left(\bar{\mu}_{t}\right)_{t}$ for $\alpha=2, \Delta t=2 \times 10^{-2}$ and $\varepsilon_{t}=0.1 /(t+1)$. 
Figure 2 shows the convergence of $\bar{\mu}_{t}$ for $\varepsilon_{t}$ satisfying $\int_{0}^{\infty} \varepsilon_{t}^{2} d t<\infty$ when $\bar{\mu}_{0}$ is chosen such that $-1<\overline{\mu_{0}} \cdot b<0$. The blue curve of Figure 2(a) corresponds to the component of $\bar{\mu}_{t}$ along the direction of $b$. We can see that the a.s. convergence of $\bar{\mu}_{t}$ to $b /|b|$ is very smooth and fast. We recover in Figure 2(b) the $L^{2}$ rate of Proposition 3. In particular, we notice that the transition phase is quite short as for $t=10$ we already observe the numerical convergence. Figure 2(c) illustrates for the same parameters the a.s. convergence rate result (see Proposition 4) for two values of $\eta$. Non surprisingly, the larger $\eta$, the smoother the convergence.

When the magnitude of the noise decays slowly, the convergence should be less smooth as suggested by Proposition 3, which corresponds to what we can see on Figure 3. Closely looking at Figures 2(a) and 3(a), we notice that the component of $\bar{\mu}$ along $b$ converges faster that the two others. Actually, from what we explained after Proposition 3 , we have

$$
\begin{aligned}
\left|\frac{b}{|b|}-\bar{\mu}_{t}\right|^{2} & =\left(1-\frac{b}{|b|} \cdot \bar{\mu}_{t}\right)+\left(\bar{\mu}_{t} \cdot e_{2}\right)^{2}+\left(\bar{\mu}_{t} \cdot e_{3}\right)^{2} \\
\left(1-\bar{\mu}_{t} \cdot e_{1}\right) & =\left(\bar{\mu}_{t} \cdot e_{2}\right)^{2}+\left(\bar{\mu}_{t} \cdot e_{3}\right)^{2} .
\end{aligned}
$$

From this last equation, it is clear that there is a power 2 difference between the rates of convergence of $\bar{\mu}_{t} \cdot b$ and of the two other components, which fully matches our numerical observations.

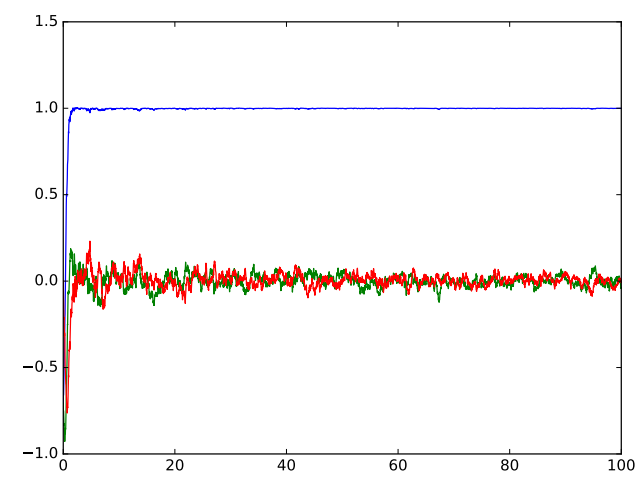

(a) A.s convergence

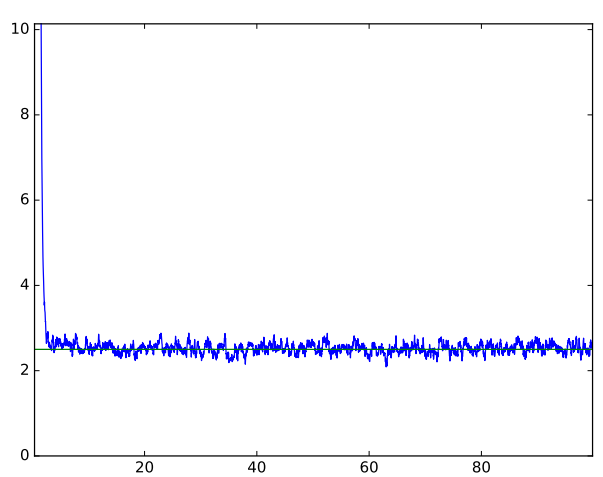

(b) Convergence rate in $L^{1}$

Figure 3: A.s. convergence of $\left(\bar{\mu}_{t}\right)_{t}$ for $\alpha=2, \Delta t=2 \times 10^{-2}$ and $\varepsilon_{t}=0.1 /(t+1)^{1 / 3}$.

From the theoretical results of Section 3 , it is clear that the noise term has a stabilizing effect on the system and is in particular responsible for escaping from $-b$, which is an unstable critical point of the deterministic system. Figure 4 confirms that the stabilizing effect exists even when the magnitude of the noise decays very fast $-\left(\varepsilon_{t}\right)_{t}$ belongs to $L^{1}([0, \infty))-$ and $\bar{\mu}_{0}=-b /|b|$, which is the worst case scenario. After a very short transition period during which $\bar{\mu}$ circles around on the sphere while heading to $b /|b|$, the process stabilizes around its limit and remains impressively smooth. 


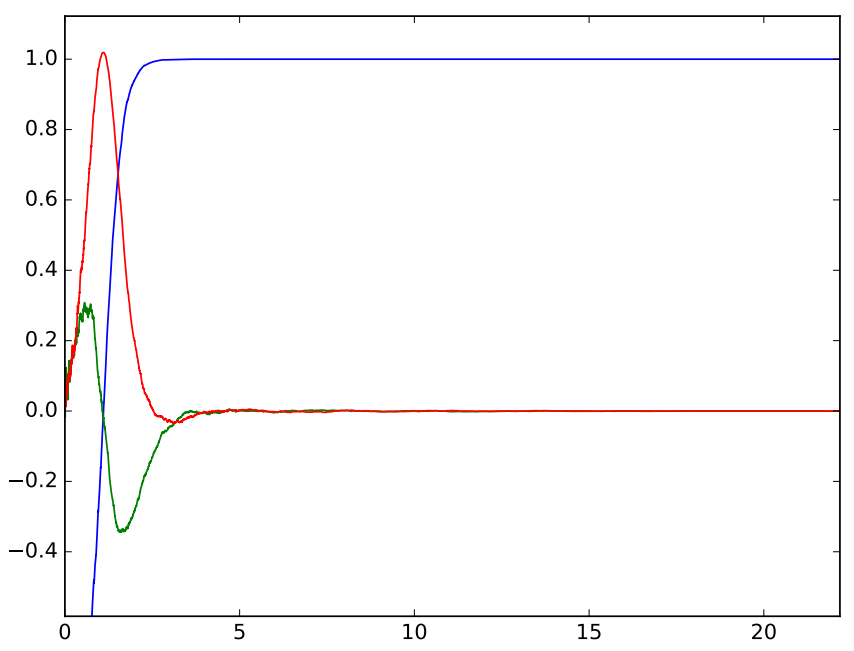

Figure 4: A.s. convergence of $\left(\bar{\mu}_{t}\right)_{t}$ for $\alpha=2, \Delta t=2 \times 10^{-3}, \bar{\mu}_{0}=-b /|b|$ and $\varepsilon_{t}=0.1 /(t+1)^{2}$.

\subsection{The case $\alpha=0$}

As emphasized by the theoretical results, the behaviour of the process $\left(\bar{\mu}_{t}\right)_{t}$ depends very much on the value of $\alpha$. When $\alpha=0$ and there is no noise, $\bar{\mu}_{t}$ evolves on a circle with constant latitude. Actually, we recover a very similar behaviour in Figure 5 when the noise magnitude decays quickly $-\int_{0}^{\infty} \varepsilon_{t} d t<\infty$. Clearly, $\bar{\mu}_{t}$ heads to a constant latitude level and keeps turning on this parallel circle but unlikely to what happens in the deterministic case, the latitude is not exactly determined by $\bar{\mu}_{0} \cdot b$ but is slightly randomly shifted as seen in Proposition 6. Closely looking at Figure 5, we can see that the amplitude of the oscillations tends to increase a little with time, which is a consequence of the discretized process not having a constant norm. This could be circumvented by considering a smaller discretization step $\Delta t$.

When the noise decreases slowly, ie. $\int_{0}^{\infty} \varepsilon_{t}^{2} d t=\infty$, its effect remains over time and prevents any almost sure limiting behaviour to appear. The process $\left(\bar{\mu}_{t}\right)$ keeps wandering around on the sphere and we see from Figure 6 that $\mathbb{E}\left[\bar{\mu}_{t}\right] \rightarrow 0$.

\section{Conclusion}

In this work, we have discussed issues on the stochastic modelling of a ferromagnetic nanoparticle. Among the different approaches, the Stratonovich approach with a decaying noise magnitude showed up as the most natural one. We investigated the long time behaviour of the model and proved its convergence to the unique stable equilibrium of the deterministic system when $\alpha>0$. When $\alpha=0$, the evolution of the system depends on the magnitude of the noise; when a limiting behaviour appears, the process keeps revolving on a parallel ring. All these theoretical results have been illustrated by numerical simulations, which help better understanding how thermal effects can be modelled in micromagnetism. 


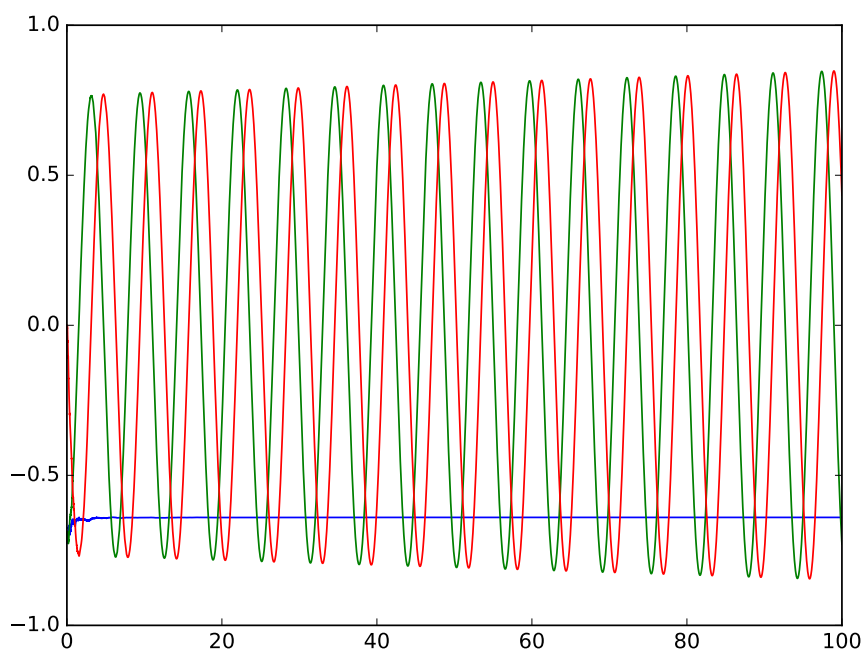

Figure 5: Convergence of $\left(\bar{\mu}_{t}\right)_{t}$ for $\varepsilon_{t}=0.3 /(t+1)^{2}, \Delta t=2 \times 10^{-3}$.

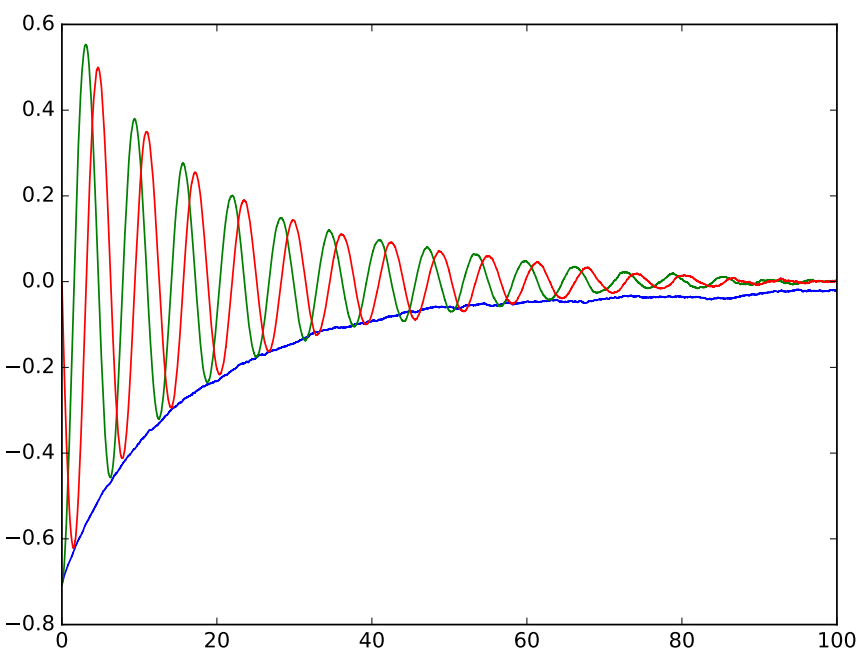

Figure 6: Convergence of $\mathbb{E}\left[\bar{\mu}_{t}\right]$ for $\varepsilon_{t}=0.3 /(t+1)^{0.1}, \Delta t=2 \times 10^{-3}$. 


\section{A Some technical lemmas}

Lemma 7. Let $F$ be a $C^{1}\left(\mathbb{R}_{+}\right)$function tending to infinity. Assume

- That there exists some $t_{0}$, such that for all $t \geq t_{0}, F(t)>0$ and $F^{\prime}(t)>0$.

- $\lim _{t \rightarrow \infty} F^{\prime}(t)=a>0$

Then for any function $g$ satisfying $\lim _{t \rightarrow \infty} g(t)=\ell$, we have

$$
\lim _{t \rightarrow \infty} \mathrm{e}^{-F(t)} \int_{0}^{t} \mathrm{e}^{F(u)} g(u)=\frac{\ell}{a} .
$$

Proof. As $F$ tends to infinity, it is clear that the integral over $\left[0, t_{0}\right]$ does not count into the final result. Considering the integral from $t_{0}$ to infinity is enough.

$F$ defines a $C^{1}$ diffeomorphism from $\left(t_{0}, \infty\right)$ on $\left(F\left(t_{0}\right), \infty\right)$. Hence, we can set the change of variable $v=F(u)$ and write

$$
\mathrm{e}^{-F(t)} \int_{t_{0}}^{t} \mathrm{e}^{F(u)} g(u)=\mathrm{e}^{-F(t)} \int_{F\left(t_{0}\right)}^{F(t)} \mathrm{e}^{v} g\left(F^{-1}(v)\right) \frac{1}{F^{\prime} \circ F^{-1}(v)} d v .
$$

$F^{-1}$ tends to infinity, hence $\lim _{v \rightarrow \infty} g\left(F^{-1}(v)\right) \frac{1}{F^{\prime} \circ F^{-1}(v)}=\frac{\ell}{a}$. Then, the result easily follows.

Lemma 8. Let $F$ be a $C^{1}\left(\mathbb{R}_{+}\right)$function tending to infinity. Assume

- there exists some $t_{0}$, such that for all $t \geq t_{0}, F(t)>0$ and $F^{\prime}(t)>0$.

- $\lim _{t \rightarrow \infty} F^{\prime}(t)=a>0$.

Then for any function $g$, we have

$$
\liminf _{t \rightarrow+\infty} \mathrm{e}^{-F(t)} \int_{0}^{t} g(u) \mathrm{e}^{F(u)} d u \geq \frac{1}{a} \liminf _{t \rightarrow+\infty} g(t) .
$$

Proof. We define $\ell=\liminf _{t \rightarrow+\infty} g(t)$. Let $\eta>0$, there exists $T>0$, such that for all $t \geq T$, $g(t) \geq \ell-\eta$

$$
\begin{aligned}
\mathrm{e}^{-F(t)} \int_{0}^{t} g(u) \mathrm{e}^{F(u)} d u & =\mathrm{e}^{-F(t)} \int_{0}^{T} g(u) \mathrm{e}^{F(u)} d u+\mathrm{e}^{-F(t)} \int_{T}^{t} g(u) \mathrm{e}^{F(u)} d u \\
& \geq \mathrm{e}^{-F(t-} \int_{0}^{T} g(u) \mathrm{e}^{F(u)} d u+\mathrm{e}^{-F(t)} \int_{T}^{t}(\ell-\eta) \mathrm{e}^{F(u)} d u .
\end{aligned}
$$

By applying Lemma 7, we get

$$
\liminf _{t \rightarrow+\infty} \mathrm{e}^{-F(t)} \int_{0}^{t} g(u) \mathrm{e}^{F(u)} d u \geq \frac{\ell-\eta}{a} .
$$

As the inequality holds for all $\eta$, the result easily follows. 


\section{References}

M. Benaim. A dynamical system approach to stochastic approximations. SIAM Journal on Control and Optimization, 34(2):437-472, 1996.

M. Benaïm and M. W. Hirsch. Stochastic approximation algorithms with constant step size whose average is cooperative. Annals of Applied Probability, 9(1):216-241, 1999.

A. Benveniste, P. Priouret, and M. Métivier. Adaptive algorithms and stochastic approximations. Springer-Verlag New York, Inc., 1990.

D. R. Brillinger. A particle migrating randomly on a sphere. Journal of Theoretical Probability, 10(2):429-443, 1997.

W.-F. Brown. Magnetostatic Principles in Ferromagnetism. North-Holland, 1962.

W. F. Brown. Thermal fluctuations of a single-domain particle. Phys. Rev., 130:1677-1686, Jun 1963. doi: 10.1103/PhysRev.130.1677.

P. Etoré, S. Labbé, and J. Lelong. Long time behaviour of a stochastic nano particle. Journal of Differential Equations, 257(6), 2014. doi: 10.1016/j.jde.2014.05.033.

J.-C. Fort and G. Pages. Asymptotic behavior of a markovian stochastic algorithm with constant step. SIAM journal on control and optimization, 37(5):1456-1482, 1999.

A. O. Ivanov, S. S. Kantorovich, V. Zverev, E. A. Elfimova, A. V. Lebedev, and A. F. Pshenichnikov. Temperature-dependent dynamic correlations in suspensions of magnetic nanoparticles in a broad range of concentrations: combined experimental and theoretical study. Phys. Chem. Chem. Phys., 2016. doi: 10.1039/C6CP02793H.

R. Khasminskii. Stochastic stability of differential equations, volume 66. Springer Science \& Business Media, 2011.

H. J. Kushner and G. G. Yin. Stochastic approximation and recursive algorithms and applications, volume 35 of Applications of Mathematics (New York). Springer-Verlag, New York, second edition, 2003. Stochastic Modelling and Applied Probability.

D. Revuz and M. Yor. Continuous martingales and Brownian motion, volume 293 of Grundlehren der Mathematischen Wissenschaften [Fundamental Principles of Mathematical Sciences]. Springer-Verlag, Berlin, third edition, 1999.

L. C. G. Rogers and D. Williams. Diffusions, Markov processes, and martingales. Vol. 2. Cambridge Mathematical Library. Cambridge University Press, Cambridge, 2000.

Y. Tserkovnyak, A. Brataas, G. E. W. Bauer, and B. I. Halperin. Nonlocal magnetization dynamics in ferromagnetic heterostructures. Rev. Mod. Phys., 77:1375-1421, Dec 2005. 